J. Phys. D: Appl. Phys., vol. 40, no. 9, pp. 2659-2665, 2007

Special cluster issue on Photonic Crystal Devices

\title{
Slow light engineering in photonic crystals
}

\author{
Toshihiko Baba and Daisuke Mori \\ Yokohama National University, Department of Electrical and Computer Engineering, \\ 79-5 Tokiwadai, Hodogayaku, Yokohama 240-8501, Japan
}

\begin{abstract}
Light showing extremely slow propagation (known as slow light) provides various effects such as spatial compression of optical signals, buffering, convolution integral calculation, beam forming, and enhancement of optical absorption, gain, nonlinearity, and so on. To generate such light, very large material or structural dispersion is used. Photonic crystal waveguides are good candidates for many device applications since they can easily generate slow light at room temperature. This paper discusses the potential of slow light devices while explaining some critical issues, such as the delay-bandwidth product and the dispersion compensation.
\end{abstract}

\section{Introduction}

The speed of light in vacuum is extremely fast $\left(c \cong 3 \times 10^{8} \mathrm{~m} / \mathrm{s}\right)$. Light can travel $30 \mathrm{~cm}$ in just 1 ns. Recently, investigations into reducing the speed of light are receiving considerable attention from many researchers, with the keyword being slow light. If it becomes possible to control the speed of light, the effect could be applied to a great variety of applications, such as delay lines that control the arrival of optical signals, optical buffers and memory to store optical signals without modification, optical calculations of the convolution integral and so on, which require a time-domain operation, and producing a light beam like an array antenna, just to name a few. In addition, slow light spatially accumulates optical signals. This can enable a great range of optical devices to be made more compact in size. At the same time, the accumulation of optical energy enhances interactions between light and matter, which can substantially enhance optical absorption, gain, phase shift, nonlinearity, and so on, per unit length.

Currently, there are two kinds of investigations being carried out, both of which are seeking to find practical applications for slow light. One uses a material dispersion and the other a structural dispersion. This paper focuses on the latter one that employs photonic crystals (PC), which have a nanostructure with multi-dimensional periodicity of the order of the wavelength of light. Special attention is paid to the fact that the optimization of PCs can overcome some major issues with slow light, including the mutually exclusive relationship between the reduction in the propagation speed and the increase in the frequency bandwidth, optical signal distortion caused by large dispersion, and reflection loss at the input/output interfaces.

\section{Generation of slow light}

When $n$ is the refractive index of the medium in which light travels, the speed of light propagation in the medium will be $c / n$. Thus, $n$ is the index indicating the deceleration of light. Note that the light assumed here is to be a continuous wave of a single frequency. For pulse-like optical signals, the group refractive index $n_{\mathrm{g}}$ is used in the place of $n$, and the velocity of optical pulses is usually expressed in terms of the group velocity $v_{\mathrm{g}} \equiv c / n_{\mathrm{g}}$. Here, $v_{\mathrm{g}}$ is given by $(d k / d \omega)^{-1}$ for the angular frequency of light $\omega$ and its wavenumber $k$. In a vacuum, $v_{\mathrm{g}}=c$ since $k=\omega / c$. In a material and/or structure which have a large first order dispersion coefficient, $v_{\mathrm{g}}$ can be greatly reduced. 
One of the phenomena which are known to generate slow light using a material dispersion is the electromagnetically induced transparency (EIT) [1], which has been investigated since the 1990s. In these investigations, a gas or solid crystal having three different and isolated electronic states is used. If a pump photon, whose energy corresponds to the energy gap from a ground state to the excited state, is injected into the medium, an excited electron coherently interacts with the photon and the excited state is split. Moreover, if signal photon having an energy corresponding to the energy gap from another ground state to the excited state is also injected, absorption occurs at two split frequencies, while transmission (i.e. no absorption) of the light occurs at the intermediate frequency (this is why the phenomenon is referred to as electromagnetically induced transparency). At the intermediate frequency, the refractive index is drastically altered by the change in the absorption in accordance with the Kramers-Kronig relations. In other words, extremely large dispersion occurs, which generates surprisingly slow light whose $v_{\mathrm{g}}$ is of the order of $\mathrm{cm} / \mathrm{s}$, i.e. $n_{\mathrm{g}}>10^{10}$ for instance [2].

Similar values of $v_{\mathrm{g}}$ are theoretically expected to be obtainable using PC waveguides, although the structural disordering of this waveguide actually limits the increase in $n_{\mathrm{g}}$ to less than 1000, as discussed later. We discuss this in detail here. PCs, which are photonic nanostructures, first came to the attention of researchers at the end of 1980s as having the potential to produce new kinds of optical devices [3] and they started to be studied worldwide from the end of 1990s [4]. These efforts resulted in the development of photonic band theory, which combines the electronic band theory and optics theory and allows the analysis of dispersion characteristics of any PC structures [5]. It has facilitated the precise design of more complex structures and optical phenomena. Moreover, the discovery of the photonic bandgap (PBG), in which the PC functions as an insulator to light of a certain frequency range, has the potential to realize some long-hoped technologies, such as nanolasers and photonic integrated circuits. Recently, there has been great progress in the area of basic technologies, as reported in many books and journal articles [6,7].

The investigations of PCs to date have employed a wide variety of different PC structures. However, the so-called PC slab, a film with a two-dimensional (2D) arrangement of holes, enjoys considerable popularity among researchers today since it is straightforward to fabricate and is very effective for those applications. In a PC slab, the total internal reflection confines light in the vertical direction, while the light propagating in the horizontal direction is dominated by the PBG. A row of line defects (defects are areas without holes) in a 2D PC slab functions as an optical waveguide [8-13]. Figure 1(a) shows a PC waveguide having this structure [14]. This example was fabricated using the simple processes of $e$-beam exposure and inductively coupled plasma etching on a silicon-on-insulator substrate. Figure 1(b) shows a photonic band for such a structure, where the horizontal axis denotes the wavenumber in the waveguide direction $k$ and the vertical axis denotes the normalized frequency $\omega a / 2 \pi c=a / \lambda$. Here, $a$ is the lattice constant and $\lambda$ is the wavelength in vacuum corresponding to the angular frequency $\omega$. The solid line indicates the photonic band of the waveguide mode and shows that light propagation occurs within the bandwidth between the band edge and the light line, which represents the condition for light to leak in the vertical direction (if there is air above and below the PC, then $\omega / c=k$ ). For the device shown in Fig. 1(a), if the slab is $200-300 \mathrm{~nm}$ thick, and $a$ is $400-450 \mathrm{~nm}$, light propagation will occur at wavelengths around $1.55 \mu \mathrm{m}$.

Here, it should be noted that the gradient of the waveguide band becomes zero at its edge. As can be seen from the formula for group velocity, $v_{\mathrm{g}}=0$ is realized on this condition. This phenomenon arises because light repeats propagating forward and backward on the Bragg condition for the PC. A similar effect is observable in any Bragg gratings and multilayer stacks. Still, due to the restriction to be described later in Section 3, light propagation is only noticeably slowed in PC waveguides and a few other photonic structures. Figure 2 shows transmission spectra obtained for an actual PC waveguide. In this spectrum, we see some fine oscillations due to Fabry-Perot resonance, whose peak-to-peak interval $\Delta \lambda$ becomes shorter towards the band edge. For the waveguide length $L, n_{\mathrm{g}}$ is given by $\lambda^{2} / 2 L \Delta \lambda$. This equation enables us to estimate $n_{\mathrm{g}}$. 
As shown in Fig. 2, $n_{\mathrm{g}}$ exceeds 100 near the band edge. Several researchers have already reported similar slow light phenomena [15-22]. Slow light is difficult to observe, however, when $n_{\mathrm{g}}$ becomes larger than this, mainly as a result of the decrease in transmission, in combination with the issues discussed below.

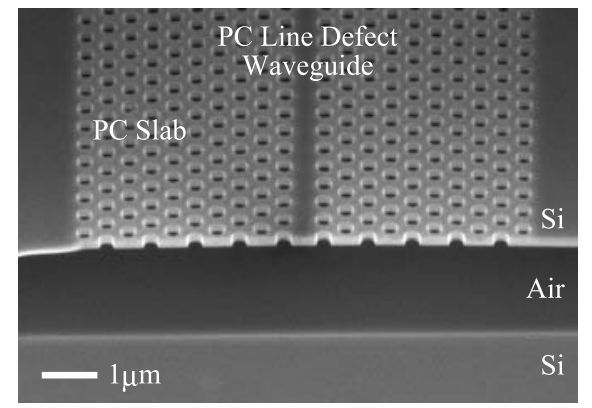

(a)

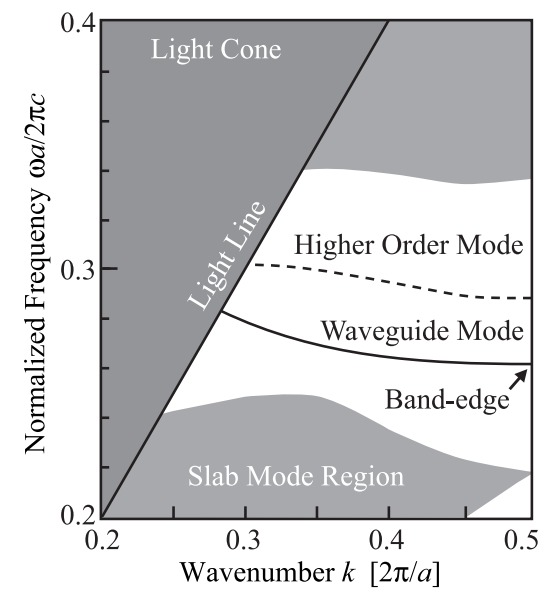

(b)

Fig. 1 PC waveguide and photonic bands. (a) Waveguide fabricated on SOI substrate. (b) Photonic band.
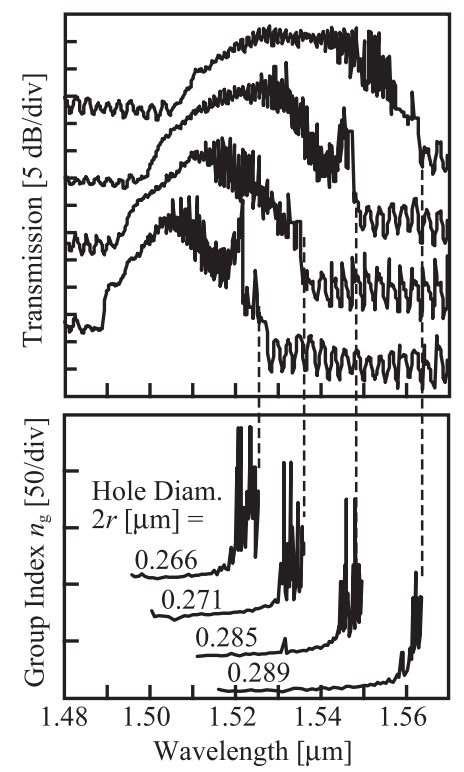

Fig. 2 Transmission spectra measured for samples having a variety of hole diameters (above), and spectra for samples having different group refractive indices (below). 


\section{Issues with slow light}

Although PCs and EIT generate slow light based on completely different principles, both of them are based upon anomalous dispersion characteristics. For this reason, these two methods share similar issues.

First, according to the formula for the group velocity, $n_{\mathrm{g}}$ is given by:

$$
n_{\mathrm{g}}=c \frac{d k}{d \omega}=\frac{d(n \omega)}{d \omega}=n+\omega \frac{d n}{d \omega}
$$

where $n$ represents the refractive index of a material in the case of EIT, and the equivalent refractive index of the guided mode for a PC waveguide. If $n_{\mathrm{g}}$ is much larger than $n$, then:

$$
n_{\mathrm{g}} \cong \frac{\Delta n}{\Delta \omega / \omega}
$$

Provided that $\Delta n$ as a fixed value, the normalized bandwidth $\Delta \omega / \omega$ must be narrow for $n_{\mathrm{g}}$ to be extremely large. For instance, if $\Delta n=0.1$, the maximum value of $n_{\mathrm{g}}$ is expected to be 485 for an optical signal bandwidth of $40 \mathrm{GHz}$ at $\lambda=1.55 \mu \mathrm{m}$ (i.e. a frequency of $194 \mathrm{THz}$ ). Also, $n_{\mathrm{g}}>10^{10}$ for EIT is only obtainable in a bandwidth of narrower than $1.9 \mathrm{kHz}$. In other words, the slowdown factor and the usable bandwidth are mutually exclusive. Changing $\Delta n$ naturally changes $n_{\mathrm{g}}$ as well. PC waveguides and EIT are designed to increase $\Delta n$. Let us suppose that there is a photonic band having a constant and very small gradient, and assume that its normalized frequency $\omega a / 2 \pi c$ is 0.25 , which is a typical value for PC waveguides. Then, within the range between the band edge and the light line of air, $\Delta n$ would be equal to 1 . However, for an actual band, like the one depicted in Fig. 1(b), $\Delta n$ becomes a fraction of 1 and, thus, values for $n_{\mathrm{g}}$ mentioned above are reasonable. We mentioned earlier in Section 2 that $v_{g}=0$ at the any band edge in a Bragg reflection structure. In usual structures, such as multilayer optical films with a small variation in refractive index and a diffraction grating with shallow grooves, $\Delta n$ is extremely small. For this reason, $n_{\mathrm{g}}$ becomes much smaller for the same $\Delta \omega / \omega$.

Another issue to overcome is the signal distortion caused by dispersion. Generally, the second order dispersion (also known as group velocity dispersion (GVD)), in which $v_{\mathrm{g}}$ varies with frequency, distorts signal waveforms. Few discussions of this issue have been published in the literature with regard to EIT since the GVD becomes zero for the intermediate frequency of the split states. By contrast, because PC waveguides have guided modes which have a curved photonic band, as shown in Fig. 1(b), large GVD occurs in the vicinity of the band edge, sometimes of the order of $100 \mathrm{ps} / \mathrm{nm}-\mathrm{mm}$ for instance, up to $10^{6}$ times larger than that in optical fibers. Both in experiments and computer simulations, attempting to reduce the propagation speed of ultra-short pulses having a full width at half maximum (FWHM) of 1 ps or shorter results in terribly deformed pulses, the original waveforms of which are almost unrecognizable at the output end of the device [17,19].

We will briefly mention further remaining issues associated with the generation of slow light. At a boundary at which $n_{\mathrm{g}}$ is drastically increased by the structural dispersion, the modal mismatch between the incident wave with a Gaussian-like profile and guided wave usually causes the strong reflection loss. The dramatic reduction in the transmittance towards the band edge shown in Fig. 2 is partly a consequence of this decrease in the transmission. Although some structural optimization of the interface structure has been discussed for PC waveguides [23,24], it is generally difficult to completely suppress the loss particularly in the slow light regime. In the case of EIT, an ultra-low temperature of below $1 \mu \mathrm{K}$ is required to stabilize the energy states of electrons. In addition, this method requires that the pump light and the signal light perfectly match the energy gap frequencies. Thus, the equipment required to generate slow light can be very large and complex. The PC waveguide can generate slow light at room temperature and consequently slow light can be generated in a compact, solid-state device, which is a major advantage of this method. In the PC waveguide, however, a subtle disordering in the structure can 
drastically increase the propagation loss of slow light. This partly explains the sudden decrease in the transmission near the band edge, as shown in Fig. 2. As a result, the highest $n_{\mathrm{g}}$ observed to date is limited of the order of several hundreds. To solve this problem, in addition to exploring the causes of such a loss and how to reduce it [25-28], it is necessary to consider a PC optical amplifier $[29,30]$, which either externally amplifies the output intensity or can be integrated with the slow light device.

\section{Wideband operation, dispersion compensation and low reflection}

Though the bandwidth restriction described in Section 3 is difficult to overcome without using a dynamic process, as described in Sec. 6, optimizing the dispersion-compensated slow light for a desired bandwidth would be very useful for many different applications. The discussion given below will explain how to realize this in a PC waveguide.

Figure 3 schematically illustrates the smooth shifting of the band edge using a PC waveguide having a chirp structure. Chirp structure refers to the continuous variation in the structural parameters along the waveguide [14]. Here, the variation is assumed to be sufficiently slow so that the periodicity of the structure and photonic band can be locally defined. Shifting the band edge this way, it is possible to convert each frequency component of an optical signal into slow light at different locations in the PC waveguide. Note that this figure does not depict a real band edge in an actual PC waveguide, but rather an ideal band for dispersion compensation, which has both zero group velocity and an inflection point [31]. If we shift such a band by chirping, the light will initially propagate with negative (or positive) dispersion. It will then be converted into slow light when it enters the region satisfying the conditions of zero group velocity. Then, when it leaves this region and starts to propagate normally again, it will be positively (or negatively) dispersed. Overall, the dispersion is compensated and light is outputted. In addition, in a chirped structure, $n_{\mathrm{g}}$ decreases gradually from the position of the zero $v_{\mathrm{g}}$, reaching almost $n_{\mathrm{g}} \sim n$ at the end of the chirped area. This also has the effect of suppressing the reflection loss mentioned in Section 3 .

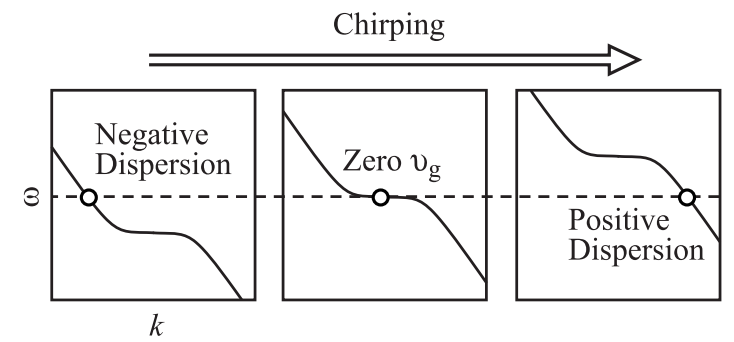

Fig. 3 Schematic band diagrams showing the shifting of an ideal band using a chirped structure.

Such an ideal band can be realized by the structural modification of the PC waveguide. (The photonic band theory is very useful for accurate and flexible design of such structures). Specifically, there are two possible ways that the PC structure can be modified. The first method uses a coupled waveguide consisting of PC waveguides of the same kind [31]. Figure 4(a) shows its example structure based on a PC directional coupler. In such a structure, two photonic bands appear, each corresponding to even- and odd-symmetric guided modes, as shown in Fig. 4(b). Increasing the area of central holes in the directional coupler forms an ideal band, as mentioned above, for an even-symmetric mode. It is necessary to split light from a single waveguide and inject the two waves having the same phase into the directional coupler. By selective use of the even mode, the desired slow light can be generated. To distinguish this method from the second one, we refer to such a device as a coupled waveguide. In this method, $\Delta n$ is slightly smaller than that by the second method. One important difference is that reflection loss, which is a serious problem in the third method, is almost negligible in the second method. The second method uses 
a directional coupler consisting of PC waveguides of different kinds having opposite dispersion characteristics [32]. Here, the zero $v_{\mathrm{g}}$ at the band edge of the PC waveguide is utilized, and the dispersion is compensated by the opposite characteristics, which make an equivalent inflection point at the band edge. One drawback is that, unless the band edges of the two waveguides perfectly match each other, reflection loss that differs from that mentioned in Section 3 will occur due to a small mode gap at the band edge and distortion of photonic bands near the band edge.

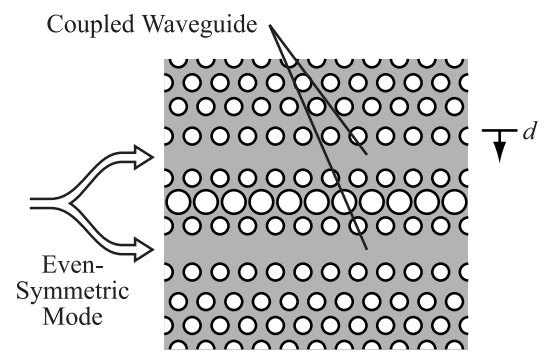

(a)

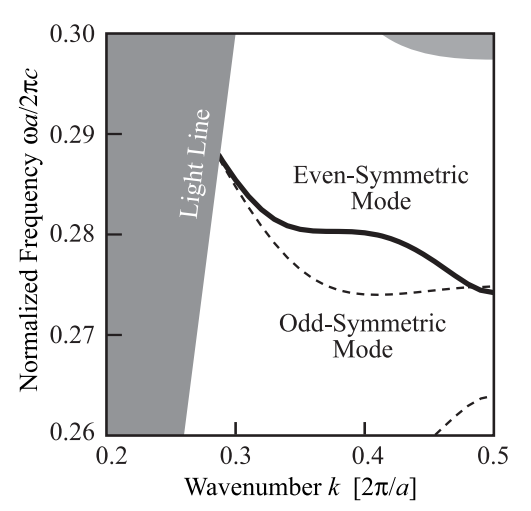

(b)

Fig. 4 PC coupled waveguide for generating slow light. (a) An example of its structure. (b) A photonic band.

Figure 5 shows chronologically the propagation of slow light calculated for the first structure shown in Fig. 4 for the case of chirping the background index [31]. The light pulse is injected into the device and is converted into slow light at the center of the device, with its waveform undeformed in the direction of propagation. In this example, because of the time limitation in performing the calculation, an ultrashort pulse having a broad frequency spectrum $(>2 \mathrm{THz})$ and rapid and drastic chirping were employed, resulting in a time delay of the order of a picosecond. However, as will be discussed below, by limiting the bandwidth appropriately and optimizing the chirping so that the device length $L$ is longer than several hundreds of microns, it is possible to generate a delay of the order of nanoseconds. Also note that in this calculation the intensity of the reflected light is suppressed to around $-30 \mathrm{~dB}$. This is due to the adiabatic transition of the light caused by the chirping.

The method described in Figs. 3-5 realizes dispersion-compensated slow light. A similar result is expected by the butt-coupling of PC waveguides having opposite dispersion characteristics [33]. Here, the operating bandwidth for $\log v_{\mathrm{g}}$ and dispersion compensation are limited, and the reflection loss at the butt-coupling has to be carefully reduced. Another approach for the dispersion issue is the generation of a dispersion-free photonic band by fine adjustment of the waveguide parameters such as enlargement of the hole diameters of a PC slab and the narrowing of line defects $[34,35,21,22]$. In this case, interactions with another band distort the guided mode resulting in a dispersion-free straight band. Because the shape of optical pulses is 
completely preserved in the waveguide, this slow light is advantageous for nonlinear applications that require a constant and high-intensity optical pulse.

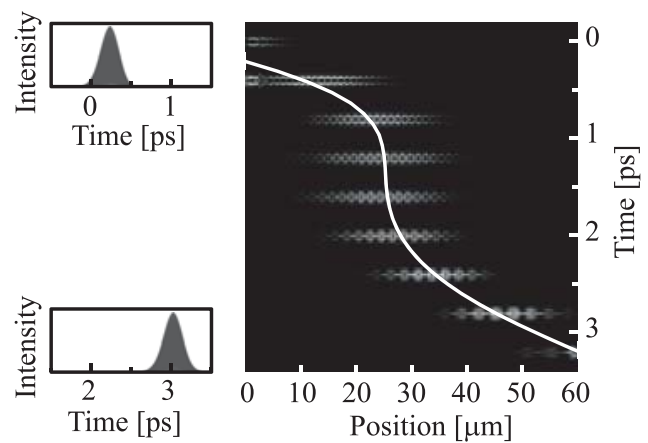

Fig. 5 Calculated results of slow light propagation in PC coupled waveguide. Reference [32] gives more details concerning calculation parameters.

\section{Average group index in chirped structure}

If $x$ is the coordinate in the direction of the propagation, the slow light time delay $T$ is given by the following equation:

$$
T=\int_{0}^{L} \frac{d x}{\mathrm{v}_{\mathrm{g}}(x)}=\int_{0}^{L} \frac{n_{\mathrm{g}}(x)}{c} d x
$$

The average group index within the chirping range $\widetilde{n}_{\mathrm{g}}$ is given by

$$
\widetilde{n}_{\mathrm{g}}=\frac{C}{\widetilde{\mathrm{v}}_{\mathrm{g}}}=\frac{C}{L / T}=\int_{0}^{L} n_{\mathrm{g}}(x) d x / L
$$

When the index chirping is assumed, the band shifts without changing its shape. On this condition, the shift of the band is considered in terms of tracing the shape of a single band. Thus, Eq. (4) can be rewritten as [32]

$$
\widetilde{n}_{\mathrm{g}} \cong \int_{\omega_{0}}^{\omega_{0}+\Delta \omega} n_{\mathrm{g}}(\omega) d \omega / \Delta \omega=\int_{\omega_{0}}^{\omega_{0}+\Delta \omega} c \frac{d k}{d \omega} d \omega / \Delta \omega=\int_{k_{0}}^{k_{0}+\Delta k} c d k / \Delta \omega=c \frac{\Delta k}{\Delta \omega},
$$

where $\omega_{0}$ and $k_{0}$ are, respectively, the frequency and the wavenumber for the point where the chirping starts. Equation (5) shows that $\widetilde{n}_{\mathrm{g}}$ is determined by the gradient of the line between the starting and ending points of the chirping, irrespective of the detailed shape of the band. This equation agrees exactly with Eq. (2), showing that the chirp structure is also subject to the bandwidth restriction.

Figure 6(a) shows the even mode bands obtained when $d$, the parameter indicating the hole position, is varied in the structure shown in Fig. 4. An ideal band is obtained when $d=0.25 a$. When $d$ exceeds this value, the gradient of the band increases, and when $d$ is smaller than this value, the band undulates. When compensating for dispersion by positive and negative dispersion within a chirp structure, the available signal bandwidth is less than half of $\Delta \omega$. To investigate this further, we computed the relationship between the normalized signal bandwidth $\Delta \omega / 2 \omega$ and $\widetilde{n}_{\mathrm{g}}$, as shown in Fig. $6(\mathrm{~b})$. When $d=0.25 a$, which gives an ideal band, narrowing the bandwidth makes $\widetilde{n}_{\mathrm{g}}$ diverge. When $\lambda=1.55 \mu \mathrm{m}$ and the signal bandwidth is $40 \mathrm{GHz}, \widetilde{n}_{\mathrm{g}}=450$. This results in a time delay of $1 \mathrm{~ns}$ for a device with $L=670 \mu \mathrm{m}$. Also, when $d$ is changed by $0.1 a$, $\widetilde{n}_{\mathrm{g}}$ decreases to almost $1 / 10$ of its optimal value. Since some local changes in the refractive index also give similar changes, it should be possible to tune $T$. 


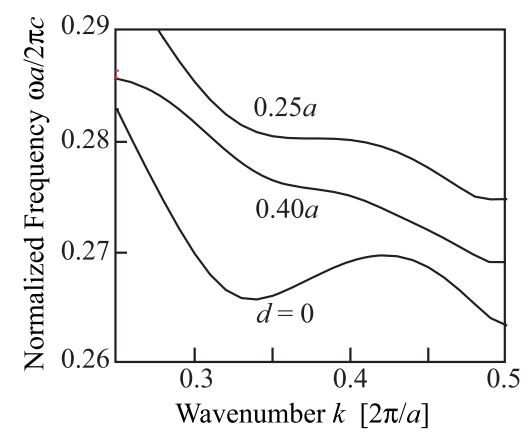

(a)

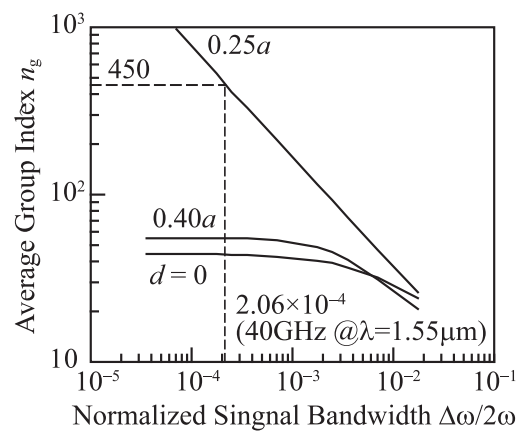

(b)

Fig. 6 Band and group index for even-symmetrical mode in PC coupled waveguide. (a) Variation in the band as a function of $d$. (b) Relationship between average group index and normalized signal bandwidth.

\section{Slow light by dynamic index modulation}

The discussion thus far has assumed that the frequency spectrum of input light does not vary with time. If a recoverable method is used for transforming a spectrum into an almost a single frequency, a fairly large $n_{\mathrm{g}}$ can be obtained in accordance with Eq. (2). Researchers are currently searching for such a method to use with both EIT and PCs.

For instance, with EIT [36], it may be possible to broaden the splitting of the excited state by enhancing the interaction of the pump light in advance and introducing signal light having broad spectrum into the medium. While $n_{\mathrm{g}}$ is small at this point, the splitting can be dynamically narrowed after the signals have entered the medium in order to increase $n_{\mathrm{g}}$. This modulates the phase of the light and adiabatically narrows the spectrum to follow the changes in dispersion. As a result of this, the signal light is converted into slow light. Also, reducing dispersion by dynamically broadening the splitting in this state recovers the spectrum as the light undergoes the inverse process.

In order to produce a similar phenomenon with a PC waveguide, some researchers have investigated changes in the dispersion caused by locally and dynamically varying the refractive index of a medium [37,38]. As explained in Section 2, a PC functions as a waveguide when a line defect is introduced. In a similar way, point defects can make the PC function as an optical cavity. If a point defect is placed in the vicinity of a line defect, the propagating light of the waveguide enters the cavity. This cavity behaves as a kind of optical memory that stores light of the resonant frequency. To make the accumulated time longer, the cavity $Q$ factor should be increased. This, however, results in a restriction to the bandwidth, as was the case with slow light. When there are many point defects located along a waveguide and the refractive index is dynamically changed between the cavity and the waveguide, changes in the dispersion characteristics can be produced. This modulates the phase of the broad spectrum of light propagating in the waveguide and 
converts it into resonant spectrum of the cavity. Also, by performing this process in the reverse order it is possible to recover the original propagating light.

These methods have the attractive advantage of overcoming the bandwidth restriction of slow light. In such methods, however, the propagating light is divided between the respective point defects and stored in them. The envelope function of the light intensity preserves the spatial distribution of the original signals. In other words, spatial compression, a phenomenon expected for slow light, does occur. For this reason, a long device is required to accumulate continuous signals. For instance, in a medium which has $n=3$, a single cycle of a $40-\mathrm{GHz}$ optical signal has a spatial length of $2.5 \mathrm{~mm}$. Supposing a single cycle corresponds approximately to a single bit, a 1-cm-long device can only accumulate a maximum of 4 bits. A further problem is that, once the refractive index has been changed, a different signal cannot be introduced. Thus, this method is suitable for the accumulation of bit-by-bit optical information similar to the method employed by dynamic random access memory.

\section{Conclusion}

We have discussed the principles of slow light, bandwidth restriction and other current issues, and possible ways to overcome these issues. Photonic crystal waveguides provide an easy way to generate slow light at room temperature. When combined with a chirp structure and a structure that compensates for dispersion, they allow the bandwidth and the waveform to be adjusted and reflection to be suppressed. For a signal bandwidth of $40 \mathrm{GHz}$, for example, it should be possible to achieve an average group velocity which is $1 / 450$ that in vacuum and a corresponding spatial compression of signals having a time delay of $1.5 \mathrm{~ns} / \mathrm{mm}$. Also, local modification of the refractive index is expected to enable the tuning of the time delay. Meanwhile, while dynamic variation of the refractive index can overcome the bandwidth restriction, such variation also causes spatial limitations. In the applications of slow light, it will be of essential to strike a good balance between slowness, bandwidth and space, according to requirements of the application.

\section{Acknowledgments}

This work was supported from the Grant-in-Aid, the IT Program and the 21st Century COE Program of Ministry of Education, Culture, Sports, Science and Technology, from the Grant-inAid and the Research Fellowship of Japan Society for the Promotion of Science, and by The Kurata Memorial Hitachi Science and Technology Foundation, The Telecommunications Advancement Foundation, and SCAT Technology Research Foundation

\section{References}

[1] See e.g., review paper Lukin M D and Imamoğlu A 2001 Nature 413273

[2] Longdell J J, Fraval E, Sellars M J and Manson N B 2005 Phys. Rev. Lett. 95063601

[3] See e.g., special issue on development and application of materials exhibiting photonic bandgaps in 1993 J. Opt. Soc. Am. B 101993

[4] See e.g., special issues on photonic crystals in 1999 J. Lightwave Technol. 17 and 2002 IEEE J. Quantum Electron. 38724

[5] See e.g., Joannopoulos J D, Meade R D and Winn J N 1995 Photonic Crystals: Molding the Flow of Light: Princeton Univ. Press

[6] See e.g., Noda S and Baba T Eds 2003 Roadmap on Photonic Crystals : Kluwer Academic

[7] Int. Workshop on Photonic and Electromagnetic Crystal Structures 1999-2005

[8] Baba T, Fukaya N and Yonekura J 1999 Electron. Lett. 35654

[9] Chutinan A and Noda S 2000 Phys. Rev. B 62, 4488

[10] Johnson S G, Villeneuve P R, Fan S and Joannopoulos J D 2000 Phys. Rev. B 628212

[11] Loncăr M, Nedeljković D, Doll T, Vučković J, Scherer A and Pearsall T P 2000 Appl. Phys. Lett. 77 1937 
J. Phys. D: Appl. Phys., vol. 40, no. 9, pp. 2659-2665, 2007

[12] Notomi M, Shinya A, Yamada K, Takahashi J and Yokohama I 2001 Electron. Lett. 37293

[13] Baba T, Motegi A, Iwai T, Fukaya N, Watanabe Y and Sakai A 2002 IEEE J. Quantum Electron. 38 743

[14] Baba T, Mori D, Inoshita K and Kuroki Y 2004 IEEE J. Sel. Top. Quantum Electron. 10484

[15] Notomi M, Yamada K, Shinya A, Takahashi J, Takahashi C and Yokohama I 2001 Phys. Rev. Lett. 87253902

[16] Asano T, Kiyota K, Kumamoto D, Song B-S, and Noda S 2004 Appl. Phys. Lett. 844690

[17] Gersen H, Karle T J, Engelen R J P, Bogaerts W, Korterik J P, van Hulst N F, Krauss T F, and Kuipers L 2005 Phys. Rev. Lett. 94073903

[18] Vlasov Y A, O’Boyle M, Hamann H F, McNab S J 2005 Nature 43865

[19] Engelen R J P, Sugimoto Y, Watanabe Y, Korterik J P, Ikeda N, van Hulst N F, Asakawa K and Kuipers L 2006 Opt. Exp. 141658

[20] Finlayson C E, Cattaneo F, Perney N M B, Baumberg J J, Netti M C, Zoorob M E, Charlton M D B and Parker G J 2006 Phys. Rev. E 73016619

[21] Frandsen L H, Lavrinenko A V, Fage-Pedersen J and Borel P I 2006 Opt. Exp. 149444

[22] Settle M D, Engelen R J P, Salib M, Michaeli A, Kuipers L and Krauss T F 2007 Opt. Exp. 15219

[23] Yamada H, Chu T, Ishida S and Arakawa Y 2005 Proc. SPIE 6019633

[24] Vlasov Y A and McNab S J 2006 Opt. Lett. 3150

[25] Gerace D and Andreani L C 2004 Opt. Lett. 291897

[26] Kuramochi E, Notomi M, Hughes S, Shinya A, Watanabe T and Ramunno L 2005 Phys. Rev. B 72 161318

[27] Hughes S, Ramunno L, Young J F, Sipe J E 2005 Phys. Rev. Lett. 94033903

[28] Johnson S G, Povinelli M L, Soljacic M, Karalis A, Jacobs S and Joannopoulos J D 2005 Appl. Phys. B 81283

[29] Raineri F, Vecchi G, Cojocaru C, Giacomotti A, Seassal C, Letartre X, Viktorovitch P, Raj R and Levenson J A 2005 Appl. Phys. Lett. 8609111

[30] Mizuta E, Watanabe H and Baba T 2006 Jpn. J. Appl. Phys. 456116

[31] Mori D and Baba T 2005 Opt. Exp. 139398

[32] Mori D and Baba T 2004 Appl. Phys. Lett. 851101

[33] Povinelli M L, Johnson S G and Joannopoulos J D 2005 Opt. Exp. 137145

[34] Sakai A, Kato I, Mori D and Baba T 2004 IEEE/LEOS Annual Meet. ThQ5

[35] Petrov A Y and Eich M 2004 Appl. Phys. Lett. 854866

[36] Li C, Dutton Z, Behroozi C H and Hau L V 2001 Nature 409490

[37] Yanik M F, Suh W, Wang Z and Fan S 2004 Phys. Rev. Lett. 93233903

[38] Notomi M, Taniyama H, Mitsugi S and Kuramochi E 2006 Phys. Rev. Lett. 97023903 